\title{
Several domains from VAR2CSA can induce Plasmodium falciparum adhesion-blocking antibodies
}

\author{
Ali Salanti, Mafalda Resende, Sisse B Ditlev, Vera V Pinto, Madeleine Dahlbäck, Gorm Andersen, Tom Manczak, \\ Thor G Theander, Morten A Nielsen
}

\begin{abstract}
Background: Malaria caused by Plasmodium falciparum can result in several different syndromes with severe clinical consequences for the about 200 million individuals infected each year. During pregnancy, women living in endemic areas become susceptible to malaria due to lack of antibodies against a unique $P$. falciparum membrane protein, named VAR2CSA. This antigen is not expressed in childhood infections, since it binds chondroitin sulphate A (CSA) expressed on the intervillous space in the placenta. A vaccine appears possible because women acquire protective antibodies hindering sequestration in the placenta as a function of parity. A challenge for vaccine development is to design small constructs of this large antigen, which can induce broadly protective antibodies. It has previously been shown that one domain of VAR2CSA, DBL4-FCR3, induces parasite adhesion-blocking antibodies. In this study, it is demonstrated that other domains of VAR2CSA also can induce antibodies with inhibitory activity.

Methods: All VAR2CSA domains from the 3D7 and HB3 parasites were produced in Baculovirus-transfected insect cells. Groups of three rats per protein were immunized and anti-sera were tested for surface reactivity against infected erythrocytes expressing FCR3 VAR2CSA and for the ability to inhibit FCR3CSA parasite adhesion to CSA. The fine specificity of the immune sera was analysed by VAR2CSA peptide arrays.

Results: Inhibitory antibodies were induced by immunization with DBL3-HB3 T1 and DBL1-3D7. However, unlike the previously characterised DBL4-FCR3 response the inhibitory response against DBL1-3D7 and DBL3-HB3 T1 was poorly reproduced in the second rounds of immunizations.

Conclusion: It is possible to induce parasite adhesion-blocking antibodies when immunizing with a number of different VAR2CSA domains. This indicates that the CSA binding site in VAR2CSA is comprised of epitopes from different domains.
\end{abstract}

\section{Background}

Pregnancy associated malaria (PAM) causing maternal anaemia, low birth weight and stillbirth, is a severe manifestation of Plasmodium falciparum infection [1]. PAM is caused by infected erythrocytes (IE) that sequester in the intervillous space of the placenta [2]. The ability to sequester in the vascular bed whereby the parasite avoids immune mechanisms in the spleen is a hallmark

\footnotetext{
* Correspondence: salanti@sund.ku.dk; salanti@sund.ku.dk

Centre for Medical Parasitology at Department of International Health, Immunology and Microbiology, University of Copenhagen and Department of Infectious Diseases, Copenhagen University Hospital (Rigshospitalet), Copenhagen, Denmark
}

of the particular virulence of $P$. falciparum. The IE bind host receptors on various endothelia through antigens called $P$. falciparum erythrocyte membrane protein 1 (PfEMP1). The PfEMP1 protein family, encoded by the var genes, is constituted of large proteins of 150-350 $\mathrm{kDa}$ with several Duffy-binding-like (DBL) domains. Different PfEMP1 molecules have different receptor specificities, and clonal switching between expression of the various var gene products, in a mutually exclusive manner, allows the parasite to modify its adhesion properties accordingly (reviewed in [3]). There are about 60 copies of highly different var genes in each parasite genome 
and a high sequence variation among genomes [4-6]. Expression of different PfEMP1 variants allow the parasites to escape previously acquired antibody responses, and clinical protection occurs when a large repertoire of variant specific antibodies allows the host to control the infection [7]. After repeated infections during childhood in endemic areas the acquired repertoire of antibodies against the variant surface antigens will progressively protect against variants expressed during severe, mild and asymptomatic infections $[8,9]$. During pregnancy the parasite again escapes acquired immunity by expressing variants not encountered during childhood disease. This is mediated by parasites occupying a new niche, the developing placenta. The interaction between parasite antigens on the surface of the IE and chondroitin sulphate A (CSA) in the placenta is one of the most direct associations between binding phenotype and disease outcome in falciparum malaria. Placental parasites and parasite lines selected for CSA binding in vitro express a unique var gene named var2csa [10,11]. VAR2CSA is expressed on the surface of IE panned on CSA and on IE isolated from infected placentas $[12,13]$ and parasite clones where the var2csa gene is disrupted lose the ability to bind CSA [14]. Several domains and regions of VAR2CSA have been shown to bind CSA in vitro, however, the specificity of single VAR2CSA domain binding to CSA does not seem to be exclusive for CSA type glycans [15-18].

Women in malaria endemic areas acquire antibodies that protect against PAM as a function of parity [1]. The mechanism of protection is suggested to be based on antibodies that block binding of IE to CSA [19]. Likewise, high anti-VAR2CSA IgG levels are correlated with protection against the clinical consequences of PAM [13]. These findings suggest that it is feasible to develop a VAR2CSA-based vaccine to protect women in malaria endemic areas against PAM. A challenge for vaccine development is to define VAR2CSA constructs of a size compatible with protein-vaccine production, which elicit pan-reactive antibodies that abrogate binding of parasites in the placenta. It has previously been shown that antibodies induced against DBL6-FCR3 partially inhibited parasite binding and that this inhibitory activity was only present in serum collected during the immunization but absent in the final bleed [20]. Recently, it was shown that DBL4-FCR3 induced a broadly IgG based parasite adhesion-blocking response, which increased during the immunizations and was highly reproducible in subsequent immunizations with the same antigen [21]. These DBL4 antibodies are currently being tested for the capacity to inhibit binding to CSA in a large panel of placental parasites. It is possible that the DBL4-FCR3 antibodies do not inhibit all isolates due to sequence variation within VAR2CSA. In that case an effective PAM vaccine would need to be based on multiple VAR2CSA serotypes. The aim of this study was to define additional DBL domain, which can induce parasite adhesion-blocking antibodies in order to make a multidomain vaccine, which presumable could protect women against multiple $P$. falciparum genotypes.

Two domains, DBL1-3D7 and DBL3-HB3 T1 were shown to induce a cross-reactive inhibitory response, however, in line with the published data on DBL6-FCR3 the inhibitory response was either not sustained during the entire immunization protocol or was not reproducible.

\section{Methods}

\section{Plasmodium falciparum cultures}

Parasite culture was grown as previously described [22]. In brief, parasites were maintained in culture using 5\% haematocrit of human blood group 0+ blood in parasite medium consisting of RPMI-1640 supplemented with 25 $\mathrm{mmol} / \mathrm{l}$ sodium bicarbonate (Sigma-Aldrich), $0.125 \mu \mathrm{g} /$ $\mathrm{ml}$ gentamycin, $0.125 \mu \mathrm{g} / \mathrm{ml}$ Albumax II (Invitrogen) and $2 \%$ normal human serum. To select for VAR2CSA expression, IE were repeatedly panned on BeWo-cells. All isolates were mycoplasma negative and were regularly genotyped using nested GLURP and MSP-2 primers in a single PCR step.

\section{Protein production}

All VAR2CSA constructs were based on native var2csa and cloned from genomic parasite DNA. Control constructs were cloned from DNA from either FCR3 var1DBL3 $\gamma$ or 3D7 PF08_0141-DBL2 $\beta$. Gene fragments were cloned into the Baculovirus vector, pAcGP67-A (BD Biosciences) modified to contain the V5 epitope upstream of a histidine tag in the C-terminal end of the constructs. Linearized Bakpak6 Baculovirus DNA (BD Biosciences) was co-transfected with pAcGP67-A into Sf9 insect cells for generation of recombinant virus particles. Histidine-tagged recombinant protein was purified on $\mathrm{Ni}^{2+}$ sepharose columns from the supernatant of Baculovirus infected High-Five insect cells using an $\Delta$ KTAxpress purification system (GE-Healthcare).

\section{Rat immunizations}

Wistar rat anti-sera raised against the VAR2CSA domains from HB3 and 3D7 were produced by injection of $40 \mu \mathrm{g}$ of recombinant protein in Freund's complete adjuvant, followed by two booster injections of $40 \mu \mathrm{g}$ of protein in Freund's incomplete adjuvant at 2 1/2-week intervals. Anti-sera were collected eight days after the final boosting injection. All immunizations induced antibodies against the recombinant proteins as measured by Enzyme-Linked Immunosorbent Assay (ELISA) of the final bleed. Immunizations with the DBL3-HB3 T1 protein were done five times and six bleeds were collected. The endpoint titer of selected serum samples and IgG 
were determined by ELISA. All procedures regarding animal immunizations complied with European and national regulations.

\section{Flow cytometry and binding inhibition assay}

Flow-cytometry was used to test the reactivity of rat serum to VAR2CSA on the surface of the IE. In brief, parasite cultures were enriched to contain late trophozoite and schizont stage parasites by exposure to a strong magnetic field. Aliquots $\left(2 \times 10^{5} \mathrm{IE}\right)$ were labelled by ethidium bromide and sequentially exposed to $10 \mu \mathrm{l}$ rat serum and $1 \mu \mathrm{l}$ anti-rat IgG-FITC (Zymax, Invitrogen). Data was acquired using a FC500 flow-cytometer (Beckman Coulter). All samples relating to a particular parasite isolate were processed and analysed in a single assay. Parasite binding assays were done as described in [21]. Briefly, $2 \times 10^{5}$ tritium labelled latestage IE and $15 \mu \mathrm{l}$ rat serum or IgG in a total volume of $100 \mu \mathrm{l}$ were added in quadruplicates to wells coated with $2 \mu \mathrm{g} / \mathrm{ml}$ of the commercially available chondroitin sulphate proteoglycan (CSPG) decorin (D8428, SigmaAldrich). The binding to decorin was abrogated by soluble CSA (C9819, Sigma-Aldrich) and chondroitinase treatment. After incubation for $30 \mathrm{~min}$ at $37^{\circ} \mathrm{C}$ unbound IE were washed away by resuspension performed by a pipetting robot (Beckman Coulter). The proportion of adhering IE was determined by liquid scintillation counting on a Topcount NXT (Perkin-Elmer).

\section{Pepscan analysis}

A peptide array containing 44231 mer peptides corresponding to the extracellular part of 3D7 VAR2CSA was used for antibody binding studies [23]. The sequences of the peptides had an overlap of six residues and the purity of the peptides was expected to be $70 \%$ or higher. The peptides were synthesized with a cysteine at amino acid position 15 allowing some secondary structure. Structural modelling and mapping of residues was done as described [23].

\section{Results}

\section{Cloning and expression of VAR2CSA DBL proteins}

To identify other domains than the published DBL4FCR3 [21], which could induce inhibitory antibodies, all single domains from 3D7 VAR2CSA and HB3 VAR2CSA were cloned (Table 1). Published data on rabbit antibodies induced against all 3D7 VAR2CSA DBL domains showed that these antibodies reacted with IE [24] but failed to inhibit parasite binding (data not shown). The 3D7 VAR2CSA DBL domains were this time cloned and expressed with the same border definitions as recently presented for the FCR3 domains [21]. The HB3 parasite genome harbours two different var2csa genes ( $\mathrm{T} 1$ and $\mathrm{T} 2$ ) and both variants were cloned and expressed. However, the DBL2 T2 and the DBL6 T2 did not express in sufficient amounts for immunizations and thus these two domains were not included in the analysis (Table 1).

Reactivity of rat IgG against native VAR2CSA and capability to block parasite adhesion to CSA

The VAR2CSA proteins were used to immunize groups of three rats. It was not possible to test inhibition of 3D7 binding, since this parasite binds poorly to CSA. With regard to the HB3 parasite, this strain harbours two variants of var2csa in the genome and panning on CSA results in expression of both var2csa variants. Therefore, all sera were evaluated on the heterologous and strongly CSA adhering parasite strain, FCR3CSA. Accordingly only cross-reactive antibodies are measured, which, in essence correspond to the response one would want from a given vaccine. All 3D7 proteins except DBL6 induced cross-reactive IgG reacting with native VAR2CSA on the surface of IE. DBL1 and DBL2 induced the highest reacting IgG (Figure 1A). The HB3 proteins induced generally poorly cross-reactive antibodies with the exception of DBL3 T1 with a MFI value of 15 (Figure 1B). The DBL5 domain has in several publications been shown to induce strong and cross-reactive IgG, however, the three different DBL5 domains produced here induced IgG, which reacted poorly with FCR3CSA (Figure 1A and 1B). The sera were tested for the ability to inhibit FCR3CSA binding to CSA. Interestingly, the DBL1-3D7 and DBL3-HB3 T1 sera had inhibitory activity and almost completely blocked the parasite adhesion to CSA (Figure 1C and 1D, respectively). None

\section{Table 1 List of immunogens. 3D7 and HB3 VAR2CSA} constructs.

\begin{tabular}{cc}
\hline 3D7 VAR2CSA single-domains & $\begin{array}{c}\text { First and last amino acid in 3D7 } \\
\text { VAR2CSA }\end{array}$ \\
\hline DBL1 & $57-437$ \\
DBL2 & $542-871$ \\
DBL3 & $1216-1571$ \\
DBL4 & $1552-1942$ \\
DBL5 & $1984-2331$ \\
DBL6 & $2313-2642$ \\
HB3 VAR2CSA Single-domains & First and last amino acid in HB3 \\
& VAR2CSA \\
DBL1 T1 & $58-403$ \\
DBL1 T2 & $58-399$ \\
DBL2 T1 & $543-901$ \\
DBL2 T2 & Not Expressed \\
DBL3 T1 & $1224-1615$ \\
DBL3 T2 & $1213-1601$ \\
DBL4 T1 & $1584-1964$ \\
DBL4 T2 & $1598-1986$ \\
DBL5 T1 & $2031-2356$ \\
DBL5 T2 & $2031-2338$ \\
DBL6 T1 & Not Expressed \\
\hline
\end{tabular}


of the other HB3 proteins elicited inhibitory antibodies when comparing to the negative control sera, whereas all the other 3D7 DBL domain sera appeared to have a weak adhesion-interfering effect.

\section{Reproducibility of the inhibitory response}

To further analyse the inhibitory response induced by DBL3-HB3 T1 and DBL1-3D7 two groups of rats were immunized and the different bleeds during the immunization procedure were analysed. The rats were immunized twice before the first bleed was collected at day 21 . The sera were tested in two different assays measuring the capacity to react with native VAR2CSA on IE and for the capacity to inhibit parasite binding to CSA in vitro. The first bleed from the DBL3-HB3 T1 immunizations blocked parasite adhesion to CSA but did not stain the surface of the parasites (Figure 2A). The inhibition remained at the same level for the subsequent four bleeds, whereas the surface reactivity
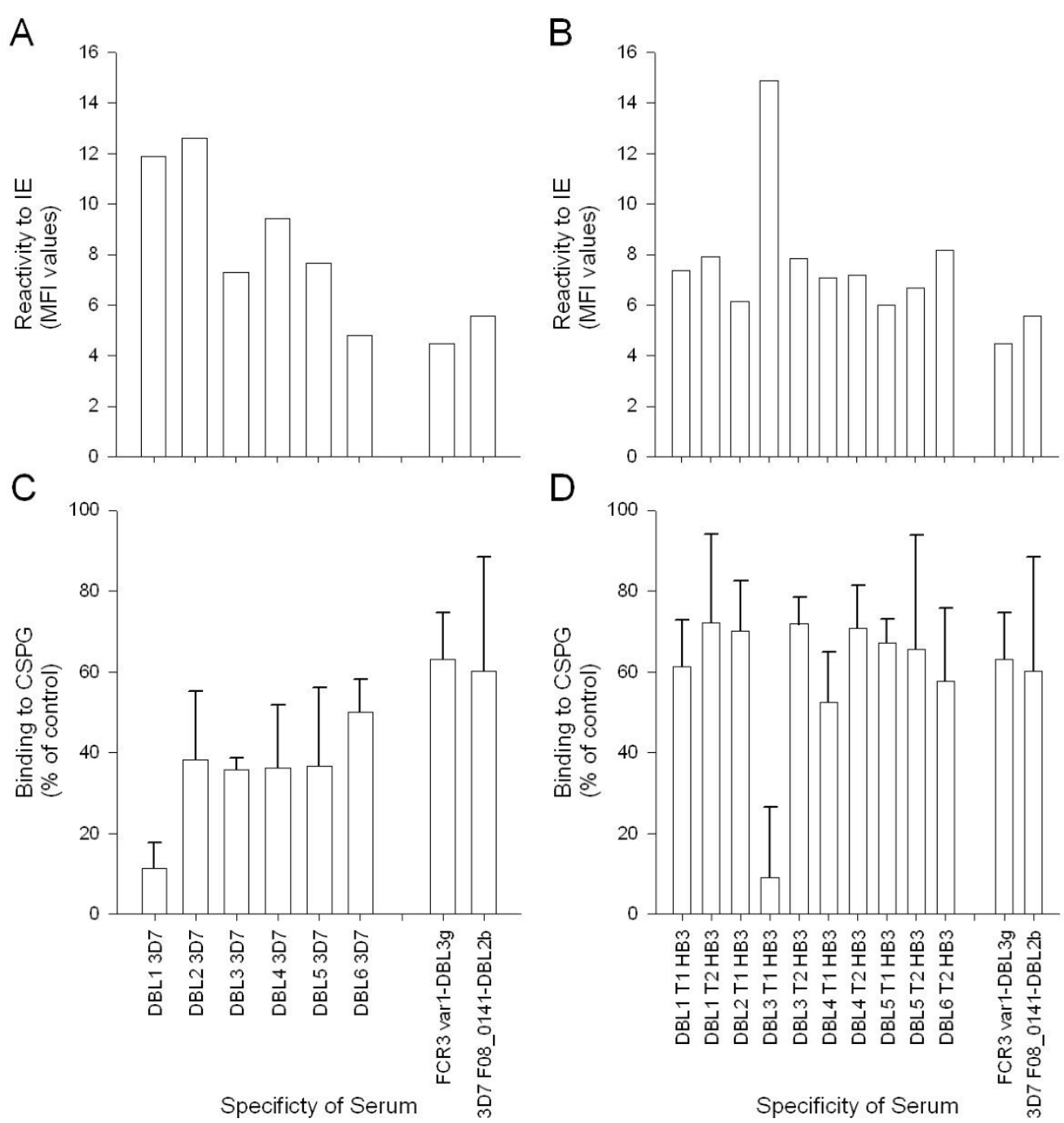

Figure 1 Surface reactivity and inhibitory capacity of serum specific for DBL domains of VAR2CSA from 3D7 and HB3. A and B: Sera from three rats immunized with the same recombinant protein were pooled and tested in FACS. The surface reactivity was measured using 10 $\mu \mathrm{l}$ serum in a total volume of $100 \mu \mathrm{l}$ against $2 \times 10^{5}$ late trophozoite and schizont-infected erythrocytes (IE). To measure the level of rat IgG bound to the surface of the IE $1 \mu \mathrm{l}$ rabbit anti-rat IgG-FITC was used in a total volume of $100 \mu \mathrm{l}$ and the mean fluorescence intensity of 5000 cells was analysed and shown in white bars. The assays were performed twice with similar results. C \& D: For inhibition of IE binding to CSPG, $15 \mu \mathrm{l}$ of rat serum in a total volume of $120 \mu \mathrm{l}$ were tested in triplicate wells of 96 well Falcon plates coated with $2 \mu \mathrm{g} / \mathrm{ml}$ CSPG. Serum pools and tritium labelled parasites $\left(2 \times 10^{5}\right)$ were added simultaneously to the wells and incubated for 2 hours. Hereafter the plate was washed using a pipetting robot and the remaining cells were harvested on a filter plate and counted using scintillation. The mean percentage of binding is shown relative to binding in wells without inhibitor. Error bars are standard deviations of triplicate measurements. The assays were performed twice with similar results. In both types of assays serum from rats immunized with FCR3 var1-DBL3 $\gamma$ and 3D7 PF08_0141-DBL2 $\beta$ were used as negative controls. 
increased. However, at the final bleed, taken 40 days after the last immunization, the IgG remained immuno fluorescence assay (IFA) positive, but no inhibitory effect was detected. In a repeated immunization experiment three new rats were immunized with DBL3-HB3 T1. This time, adhesion-blocking antibodies were not detected in any of the bleeds, whereas the IgG reactivity against FCR3CSA increased during the immunizations (Figure 2B). When repeating the DBL1-3D7 immunizations, there was no appreciable anti-adhesive effect of the serum in any of the bleeds. Only the third bleed appeared to react with the surface of FCR3CSA, although this reactivity was not present in the final bleed (Figure 2C).

\section{Epitope mapping of induced anti-VAR2CSA IgG}

The fine specificity of the antibody response in inhibitory and non-inhibitory sera was analysed using a peptide array covering the extracellular part of 3D7 VAR2CSA consisting of 442 peptides of 31 mer each with an overlap of six residues. The peptides were
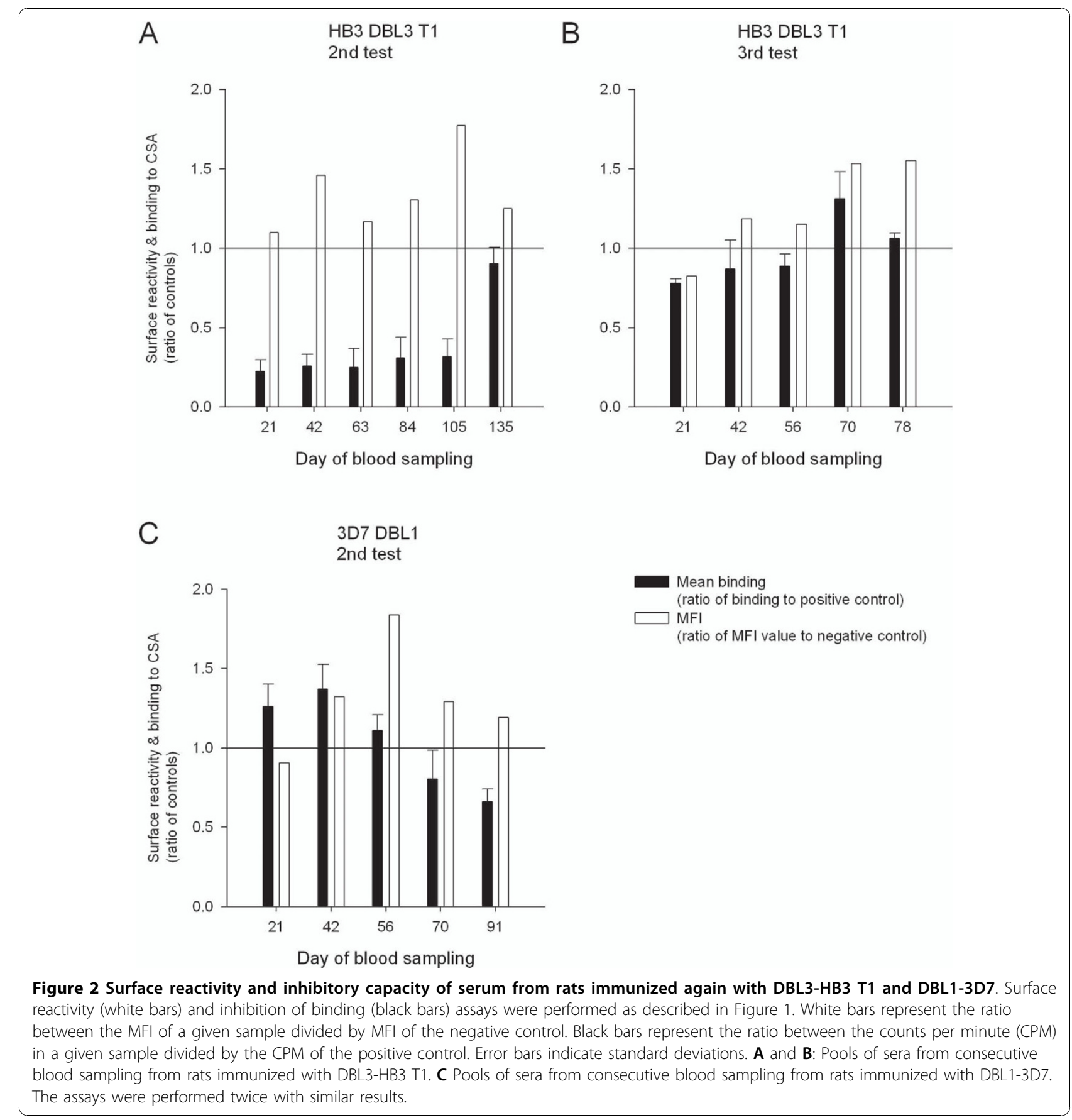
bound to the chip using a cysteine at position 15. From the DBL1-3D7 immunizations, the original inhibitory serum sample was analysed on the chip. In addition sera from two rats from the subsequent immunizations, which did not induce inhibitory antibodies (Figure 3A), were analysed. In all the sera, there was a high reactivity against eight to 17 peptides of the total 63 peptides covering the DBL1 domain and three major B-cell epitopes were detected. Two of these epitopes reacted with IgG from all three sera, of which one was located between subdomain S1 and S2 (marked with a blue horizontal bar in figure $3 \mathrm{~A}$ ) and the other one at the beginning of subdomain S2 (orange in figure 3A), whereas the third epitope was predominantly recognized by the inhibitory serum and located in subdomain S3 (red horizontal bar, figure 3A). For DBL3-HB3 T1 the IgG reactivity from two rat sera were analysed, which inhibited parasite binding, and one rat serum that did not inhibit binding (Figure 3B). Two regions at the beginning of the S2 and S3 subdomain, respectively, were targeted by IgG from all three sera. However, only one peptide within each region was recognized by the non-inhibitory serum. The inhibitory DBL4-FCR3 antibodies published recently [21] were also mapped for epitopes using the same peptide array. It was difficult to identify a DBL4-FCR3 serum sample which did not induce inhibitory antibodies, therefore, a non-inhibitory DBL4-3D7 serum sample was chosen for comparison in the pepscan analysis (Figure 3C). Similar to the antibody reactivity induced against DBL1 and DBL3, a B-cell epitope was identified in the $\mathrm{N}$-terminal part of the domain spanning the $\mathrm{S} 1$ and $\mathrm{S} 2$ subdomain border (orange horizontal bar in figure $3 \mathrm{C}$ ) as well as a B-cell epitope in S3 (blue horizontal bar in figure 3C). All identified B-cell epitope regions were mapped on a model of the corresponding domain (Figure 3A-C).

\section{Discussion}

Identification of the var2csa gene is one of the major recent advances in the search for a vaccine against PAM. The low level of polymorphism in VAR2CSA, relative to other PfEMP1 molecules [25], and the rapid acquisition of VAR2CSA-specific IgG as a function of parity after natural infections [13], support the potential of developing parts of this antigen into a vaccine. PAM pathogenesis is partly due to host immune responses in the placenta [26], and it may not be optimal to induce IgG that are not adhesion-inhibitory, since this may enhance unwanted inflammatory immune responses in the placental tissue. IgG that hinder parasite binding would reduce the harmful inflammatory response in the placenta and depending on the IgG isotype, these antibodies could also be efficiently opsonising. The strategy for vaccine development is therefore to induce anti- adhesive antibodies [27]. It has previously been shown that the DBL4 domain from FCR3 elicits a strong inhibitory IgG response, which was highly reproducible in subsequent immunizations [21]. This has raised the question whether DBL4 is the only part of VAR2CSA involved in CSA adhesion, and if other domains are indeed involved in the adhesion process, and accordingly it would be of interest to define these to make a multidomain vaccine. Interestingly, in this study it is shown that two domains different from DBL4 can also elicit inhibitory antibodies. This indicates that the CSA binding region of VAR2CSA is comprised of epitopes from multiple domains, which is in line with published data showing that single recombinant domains from VAR2CSA do not bind specifically to CSA $[15,17]$. DBL4 is the most conserved VAR2CSA domain; however this domain does contain some variable regions, primarily located in the flexible loops [28]. At present, it is not known whether the anti-DBL4 IgG can inhibit parasite adhesion of all VAR2CSA expressing isolates. Therefore, a VAR2CSA vaccine may be comprised of DBL4 from different var2csa genes or FCR3 DBL4 and another DBL domain. From a vaccine development point of view, it is thus promising that it is possible to induce highly inhibitory antibodies with other domains than DBL4-FCR3. It is surprising though that a strong inhibitory antibody response could not be induced with the DBL4 domains from 3D7 and the two variants of HB3. In addition, the induced inhibitory response towards DBL3-HB3 T1 and DBL1-3D7 appeared to be difficult to reproduce in subsequent immunizations.

Based on the above findings, it is suggested that antibodies can block a single unique CSA binding-site present in the quaternary structure of VAR2CSA, or block the assembly of such quaternary structure to interfere with binding, and that these antibodies can be induced by a number of different VAR2CSA domains. This hypothesis is in line with previously published data suggesting that VAR2CSA is presented as a globular protein on the surface of IE [23]. However, it seems difficult to focus the immune response in the animals towards the correct epitopes, which is substantiated by the fact that the inhibitory response is either lost during the immunization schedule, or not induced at all despite the presence of high reactivity in ELISA.

The binding inhibitory activity was not induced in all immunizations; despite the presence of a specific and high titered immune response. This could be due to an erratic animal response to the immunogen caused by an unstable tertiary structure of the antigens used for immunizations. Another explanation could be differences in the fine specificity of the sera affecting the functionality of the induced IgG response, due to selection for reactivity against loop regions with high 


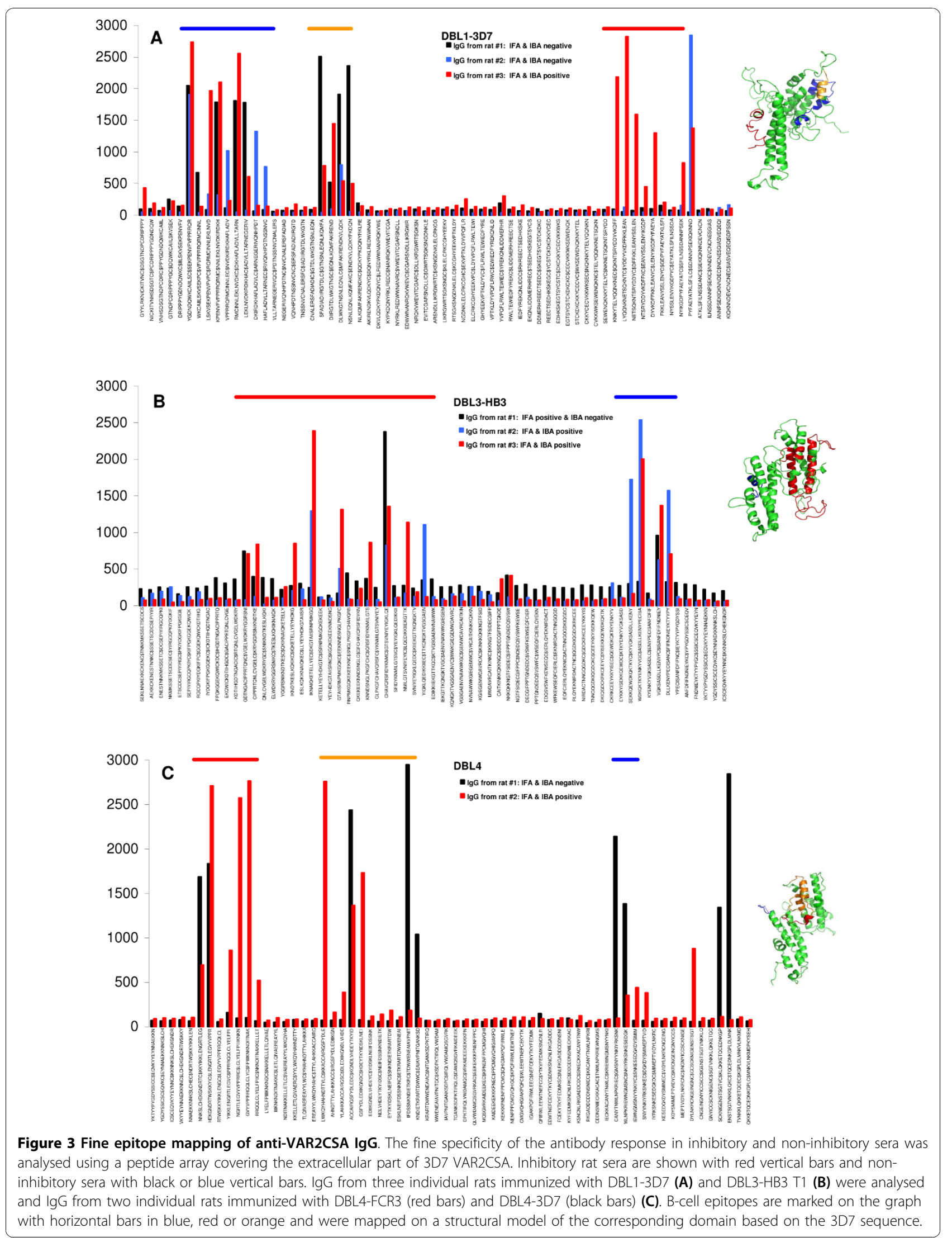


sequence variation, which this study would be sensitive to, since heterologous responses are measured. To study the fine specificity of the different sera, the IgG reactivity against VAR2CSA peptides was measured. In general, it was noted that the measured antibody reactivity against the different domains located to the same regions, and that a large central region of the domain did not contain any continuous epitopes. Interestingly, the large epitope in DBL3 located in a flexible loop is identical to the loop region shown to become structured upon CSA interaction [29]. DBL domains are compact and highly structured proteins and it is likely that many antibodies will be targeting discontinuous epitopes and thus would not be detected in the peptide array. However, it is clear from the data that different immunizations with the same antigen do not give the same fine specificity each time, which likely reflects the variation in the inhibitory activity of the induced antibodies.

\section{Conclusions}

VAR2CSA consists of six DBL domains. The data presented here show that it is possible to induce parasite adhesion-blocking antibodies when immunizing animals with the domains DBL1 and DBL3, which indicates that the CSA binding site in VAR2CSA is comprised of regions from different domains. However, a robust and reproducible response with these proteins was not inducible. Epitope mapping shows differences in the fine specificity of the inhibitory and non-inhibitory sera. Accordingly if the Baculovirus produced DBL1-3D7 and DBL3-HB3 T1 antigens are to be used in a vaccine, further research is needed to define and focus the immune response towards the relevant epitopes.

\section{Abbreviations}

CSA: chondroitin sulfate A; CSPG: chondroitin sulphate proeoglycan; DBL: Duffy-binding-like; EBA: Erythrocyte Binding Antigen; IE: infected erythrocytes; ELISA: Enzyme-Linked Immunosorbent Assay; IBA: Inhibition binding assay; IFA: Immunofluorescence assay; IgG: immunoglobulin gamma; PAM: pregnancy-associated malaria; PfEMP1: Plasmodium falciparum Erythrocyte Membrane Protein 1

\section{Acknowledgements \\ The authors would like to thank Technicians Anne Corfitz, Besim Berisha, Jonas Fjelbye Hansen, Maiken Visti and Nahla Chehabi for excellent technical assistance. This study received funding from the Danish Research Council (SSVF) (grant 22-03-0333) and the Danish Research Council for Development Research (RUF) (grant 104. Dan.8.L.306 and 8.L.306). MAN and AS are supported by the PMI2 Gates Malaria Programme grant from the Bill and Melinda Gates foundation. MD is supported by Danish Council for Independent Research/Medical Sciences (FSS) grant \#271-07-0696. The research leading to these results also received funding from the European Community's Seventh Framework Programme ([FP7/2007-2013]) under grant agreement n²01222.}

\section{Authors' contributions}

AS and MD produced the proteins and MR and SBD immunized animals. MAN and VP performed the parasite experiments. MR, MAN, TM, MD, TGT and AS were responsible for the study design and the interpretation of the data. All authors contributed to writing of the manuscript and approved the final version.

\section{Competing interests}

The authors declare that they have no competing interests.

Received: 1 October 2009

Accepted: 11 January 2010 Published: 11 January 2010

\section{References}

1. Brabin BJ: An analysis of malaria in pregnancy in Africa. Bull World Health Organ 1983, 61:1005-1016.

2. Walter PR, Garin Y, Blot P: Placental pathologic changes in malaria. A histologic and ultrastructural study. Am J Pathol 1982, 109:330-342.

3. Kraemer SM, Smith JD: A family affair: var genes, PfEMP1 binding, and malaria disease. Curr Opin Microbiol 2006, 9:374-380.

4. Baruch DI, Pasloske BL, Singh HB, Bi X, Ma XC, Feldman M, Taraschi TF, Howard RJ: Cloning the $P$. falciparum gene encoding PfEMP1, a malarial variant antigen and adherence receptor on the surface of parasitized human erythrocytes. Cell 1995, 82:77-87.

5. Smith JD, Chitnis CE, Craig AG, Roberts DJ, Hudson-Taylor DE, Peterson DS, Pinches R, Newbold Cl, Miller LH: Switches in expression of Plasmodium falciparum var genes correlate with changes in antigenic and cytoadherent phenotypes of infected erythrocytes. Cell 1995, 82:101-110.

6. Su XZ, Heatwole VM, Wertheimer SP, Guinet F, Herrfeldt JA, Peterson DS, Ravetch JA, Wellems TE: The large diverse gene family var encodes proteins involved in cytoadherence and antigenic variation of Plasmodium falciparum-infected erythrocytes. Cell 1995, 82:89-100.

7. Bull PC, Lowe BS, Kortok M, Molyneux CS, Newbold Cl, Marsh K: Parasite antigens on the infected red cell surface are targets for naturally acquired immunity to malaria. Nat Med 1998, 4:358-360.

8. Jensen ATR, Magistrado P, Sharp S, Joergensen L, Lavstsen T, Chiucciuini A, Salanti A, Vestergaard LS, Lusingu JP, Hermsen $R$, Sauerwein $R$, Christensen J, Nielsen MA, Hviid L, Sutherland C, Staalsoe T, Theander TG: Plasmodium falciparum associated with severe childhood malaria preferentially expresses PfEMP1 encoded by group A var genes. J Exp Med 2004, 199:1179-1190.

9. Rottmann M, Lavstsen T, Mugasa JP, Kaestli M, Jensen AT, Muller D, Theander T, Beck HP: Differential expression of var gene groups is associated with morbidity caused by Plasmodium falciparum infection in Tanzanian children. Infect Immun 2006, 74:3904-3911.

10. Tuikue Ndam NG, Salanti A, Bertin G, Dahlback M, Fievet N, Turner L, Gaye A, Theander T, Deloron P: High level of var2csa transcription by Plasmodium falciparum isolated from the placenta. J Infect Dis 2005, 192:331-335.

11. Salanti A, Staalsoe T, Lavstsen T, Jensen ATR, Sowa MPK, Arnot DE, Hviid L Theander TG: Selective upregulation of a single distinctly structured var gene in chondroitin sulphate A-adhering Plasmodium falciparum involved in pregnancy-associated malaria. Mol Microbiol 2003, 49:179-191.

12. Magistrado $P$, Salanti $A$, Tuikue Ndam NG, Mwakalinga SB, Resende M, Dahlback M, Hviid L, Lusingu J, Theander TG, Nielsen MA: VAR2CSA expression on the surface of placenta-derived Plasmodium falciparuminfected erythrocytes. J Infect Dis 2008, 198:1071-1074.

13. Salanti A, Dahlback M, Turner L, Nielsen MA, Barfod L, Magistrado P, Jensen AT, Lavstsen T, Ofori MF, Marsh K, Hviid L, Theander TG: Evidence for the involvement of VAR2CSA in pregnancy-associated malaria. J Exp Med 2004, 200:1197-1203.

14. Viebig NK, Gamain B, Scheidig C, Lepolard C, Przyborski J, Lanzer M, Gysin J, Scherf A: A single member of the Plasmodium falciparum var multigene family determines cytoadhesion to the placental receptor chondroitin sulphate A. EMBO Rep 2005, 6:775-781.

15. Resende M, Ditlev SB, Nielsen MA, Bodevin S, Bruun S, Pinto W, Clausen $H$, Turner L, Theander TG, Salanti A, Dahlback M: Chondroitin sulphate A (CSA)-binding of single recombinant Duffy-binding-like domains is not restricted to Plasmodium falciparum Erythrocyte Membrane Protein 1 expressed by CSA-binding parasites. Int J Parasitol 2009, 39:1195-1204.

16. Dahlback M, Rask TS, Andersen PH, Nielsen MA, Ndam NT, Resende M, Turner L, Deloron P, Hviid L, Lund O, Pedersen AG, Theander TG, Salanti A: Epitope mapping and topographic analysis of VAR2CSA DBL3X involved in P. falciparum placental sequestration. PLOS Pathog 2006, 2:e124. 
17. Khunrae P, Philip JM, Bull DR, Higgins MK: Structural comparison of two CSPG-binding DBL domains from the VAR2CSA protein important in malaria during pregnancy. J Mol Biol 2009, 393:202-213.

18. Gamain B, Trimnell AR, Scheidig C, Scherf A, Miller LH, Smith JD: Identification of multiple chondroitin sulfate A (CSA)-binding domains in the var2CSA gene transcribed in CSA-binding parasites. I Infect Dis 2005, 191:1010-1013.

19. Fried M, Nosten F, Brockman A, Brabin BJ, Duffy PE: Maternal antibodies block malaria. Nature 1998, 395:851-852.

20. Fernandez P, Kviebig N, Dechavanne S, Lepolard C, Gysin J, Scherf A, Gamain B: Var2CSA DBL6-epsilon domain expressed in HEK293 induces limited cross-reactive and blocking antibodies to CSA binding parasites. Malar J 2008, 7:170.

21. Nielsen MA, Pinto W, Resende M, Dahlback M, Ditlev SB, Theander TG, Salanti A: Induction of adhesion-inhibitory antibodies against placental Plasmodium falciparum parasites by using single domains of VAR2CSA. Infect Immun 2009, 77:2482-2487.

22. Nielsen MA, Resende M, Alifrangis M, Turner $L$, Hviid L, Theander TG, Salanti A: Plasmodium falciparum: VAR2CSA expressed during pregnancyassociated malaria is partially resistant to proteolytic cleavage by trypsin. Exp Parasitol 2007, 117:1-8.

23. Andersen P, Nielsen MA, Resende M, Rask TS, Dahlback M, Theander T, Lund O, Salanti A: Structural insight into epitopes in the pregnancyassociated malaria protein VAR2CSA. PLoS Pathog 2008, 4:e42.

24. Barfod L, Nielsen MA, Turner L, Dahlback M, Jensen AT, Hviid L, Theander TG, Salanti A: Baculovirus-expressed constructs induce immunoglobulin $\mathrm{G}$ that recognizes VAR2CSA on Plasmodium falciparuminfected erythrocytes. Infect Immun 2006, 74:4357-4360.

25. Trimnell AR, Kraemer SM, Mukherjee S, Phippard DJ, Janes JH, Flamoe E, Su XZ, Awadalla P, Smith JD: Global genetic diversity and evolution of var genes associated with placental and severe childhood malaria. Mol Biochem Parasitol 2006, 148:169-180.

26. Fried M, Muga RO, Misore AO, Duffy PE: Malaria elicits type 1 cytokines in the human placenta: IFN-gamma and TNF-alpha associated with pregnancy outcomes. J Immunol 1998, 160:2523-2530.

27. Duffy PE, Fried M: Plasmodium falciparum adhesion in the placenta. Curr Opin Microbiol 2003, 6:371-376.

28. Bockhorst J, Lu F, Janes JH, Keebler J, Gamain B, Awadalla P, Su XZ, Samudrala R, Jojic N, Smith JD: Structural polymorphism and diversifying selection on the pregnancy malaria vaccine candidate VAR2CSA. Mol Biochem Parasitol 2007, 155:103-112.

29. Higgins MK: The structure of a chondroitin sulfate-binding domain important in placental malaria. J Biol Chem 2008, 283:21842-21846.

\section{doi:10.1186/1475-2875-9-11}

Cite this article as: Salanti et al.: Several domains from VAR2CSA can induce Plasmodium falciparum adhesion-blocking antibodies. Malaria Journal 2010 9:11.

\section{Publish with Bio Med Central and every scientist can read your work free of charge}

"BioMed Central will be the most significant development for disseminating the results of biomedical research in our lifetime. "

Sir Paul Nurse, Cancer Research UK

Your research papers will be:

- available free of charge to the entire biomedical community

- peer reviewed and published immediately upon acceptance

- cited in PubMed and archived on PubMed Central

- yours - you keep the copyright 